\title{
Apathy is associated with disruption to distinct large-scale white matter networks
}

J Tay ${ }^{1}$, AM Tuladhar ${ }^{2}$, MJ Hollocks ${ }^{1}$, RL Brookes ${ }^{1}$, DJ Tozer ${ }^{1}$, TR Barrick $^{3}$, M Husain ${ }^{4}$, FE de Leeuw², HS Markus $^{\star 1}$

${ }^{1}$ Department of Clinical Neurosciences, University of Cambridge. ${ }^{2}$ Donders Institute for Brain, Cognition and Behaviour, Radboud University Nijmegen Medical Centre. ${ }^{3}$ Neuroscience Research Centre, Molecular and Clinical Sciences Research Institute, St. George's University of London. ${ }^{4}$ Nuffield Department of Clinical Neurosciences, University of Oxford.

\section{Introduction}

Apathy is a common syndrome in many neurological disorders, but its pathophysiology is poorly understood. Research has shown that apathy, but not depression, is associated with widespread white matter tract damage, implicating apathy as a disconnection syndrome. We test this hypothesis using network analysis to study white matter networks in patients with cerebral small vessel disease (SVD), a vascular pathology characterized by white matter damage and apathy.

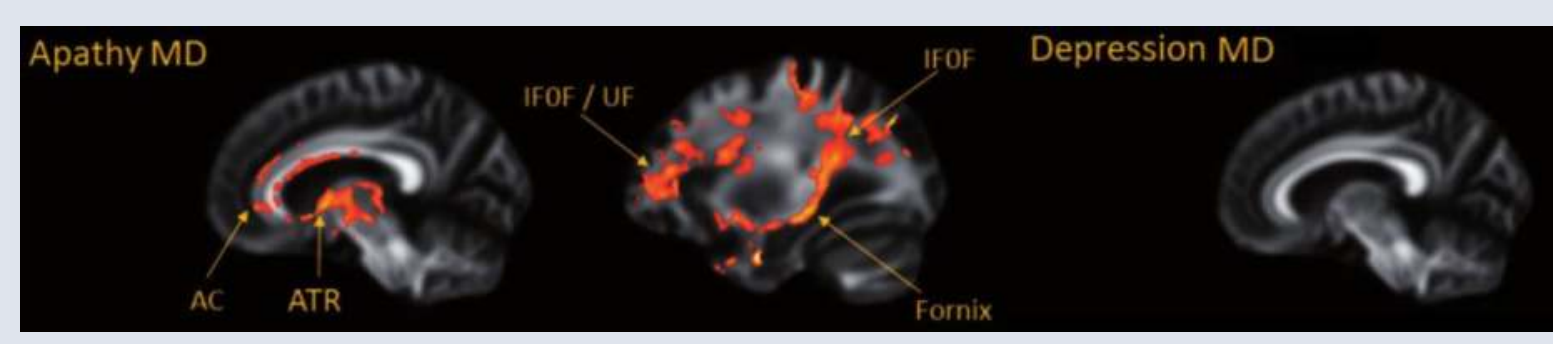

Figure 1: Apathy, but not depression, is associated with white matter tract damage. (Hollocks et al. Brain 2015)

\section{Objectives}

1) To show that network measures mediate the relationship between radiological markers of SVD and apathy.

2) To determine how white matter networks of SVD patients with apathy are disrupted.

3) To localise where apathy-related network disruption occurs.

\section{Methods}

Participants: 331 SVD patients from the RUN DMC cohort study.

Measures: Apathy: Apathy Evaluation Scale (AES); Depression: Centre for Epidemiological Studies Depression Scale (CESD).

Group assignment: Total AES and CESD scores were dichotomized based on cut scores (apathy $=A E S \geq 34$, depression $=C E S D \geq 16$ ). Subjects were then divided into four groups based on their AES and CESD scores: apathy only $(n=26)$, depression only $(n=48)$, comorbid apathy and depression $(n=32)$, and a no apathy or depression group used as a "control" ( $n=225)$.

MRI scans: Images were acquired on a Siemens Magnetom Avanto Tim 1.5T MRI. The protocol included a T1-weighted MPRAGE sequence $(\mathrm{TR}=2250 \mathrm{~ms}, \mathrm{TE}=2.95 \mathrm{~ms}, \mathrm{TI}=850 \mathrm{~ms}$, voxel size $=1.0$ $\mathrm{mm}$ isotropic) and a diffusion-weighted EPI sequence (TR $=10200$ $\mathrm{ms}, \mathrm{TE}=95 \mathrm{~ms}$, voxel size $=2.5 \mathrm{~mm}$ isotropic; 7 scans with $b=0$ $\mathrm{s} / \mathrm{mm}^{2}, 61$ scans with $\left.b=900 \mathrm{~s} / \mathrm{mm}^{2}\right)$.

Network construction: T1 images were parcellated into 90 regions (network nodes) using the Automated Anatomical Labelling atlas. Deterministic diffusion tensor tractography was used to reconstruct white matter fibres (network edges). Two nodes were considered connected if joined by an edge. This resulted in a 90x90 matrix describing connectivity between regions for each subject.

\section{Statistical analysis}

Mediation analysis: Path analysis was used to test mediation hypotheses related to SVD pathology, network measures, and apathy. Whole brain network analysis: Summary network measures were computed for each subject. Strength and density measure the quantity of connections in a network, while global and local efficiency measure the organization of those connections. These measures were then compared between groups using one-way ANOVAs and Tukey's HSD.

Regional network analysis: To determine specific nodes and edges associated with apathy, network matrices were compared between the apathy group and all other subjects using a $t$-test. We then examined edges correlated with apathy while controlling for depression in all subjects using a regression analysis.

\section{Results}

Mediation analysis

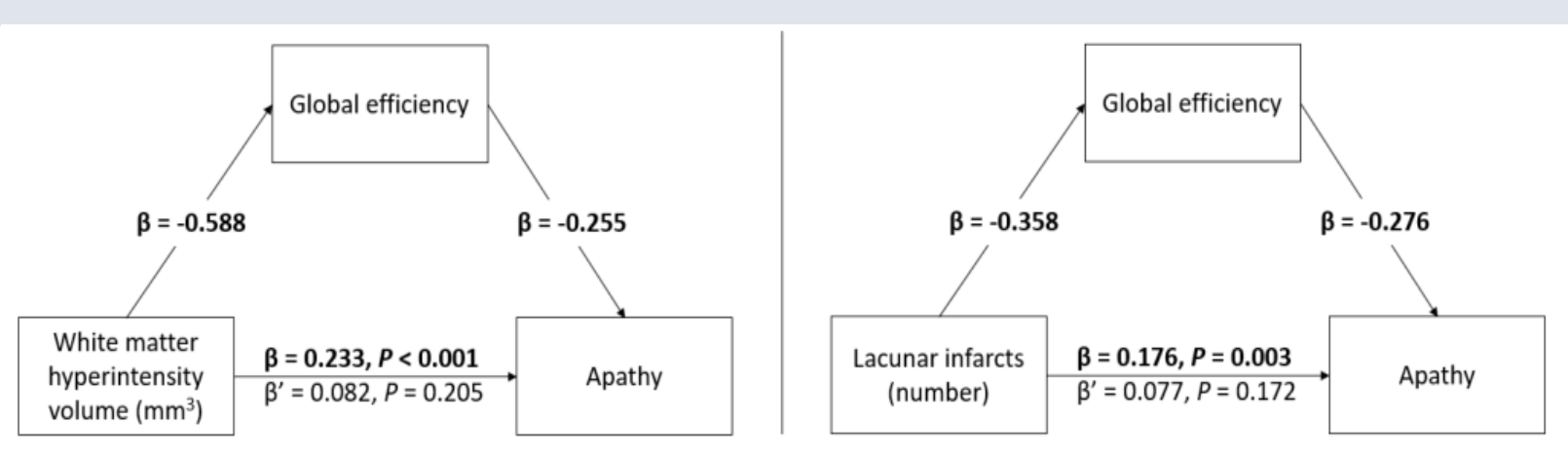

Figure 2: Global efficiency mediates the relationship between SVD pathology and apathy.

Whole brain network analysis

\begin{tabular}{|l|c|c|c|c|c|c|}
\hline $\begin{array}{l}\text { Network } \\
\text { Parameter }\end{array}$ & $\begin{array}{c}\text { Apathy - } \\
\text { depression }\end{array}$ & $\begin{array}{c}\text { Apathy - } \\
\text { comorbid }\end{array}$ & $\begin{array}{c}\text { Apathy - } \\
\text { control }\end{array}$ & $\begin{array}{c}\text { Depression - } \\
\text { comorbid }\end{array}$ & $\begin{array}{c}\text { Depression - } \\
\text { control }\end{array}$ & $\begin{array}{c}\text { Comorbid - } \\
\text { control }\end{array}$ \\
\hline Strength & $P<0.001$ & $P=0.023$ & $P<0.001$ & $P=0.708$ & $P=0.915$ & $P=0.257$ \\
\hline Density & $P<0.001$ & $P=0.010$ & $P<0.001$ & $P=0.840$ & $P=0.976$ & $P=0.530$ \\
\hline Global Efficiency & $P<0.001$ & $P=0.013$ & $P<0.001$ & $P=0.822$ & $P=0.873$ & $P=0.333$ \\
\hline Local Efficiency & $P=0.003$ & $P=0.079$ & $P<0.001$ & $P=0.757$ & $P=0.950$ & $P=0.356$ \\
\hline
\end{tabular}

Table 1: Post-hoc between-group comparisons on whole brain network measures.

Regional network analysis

A)

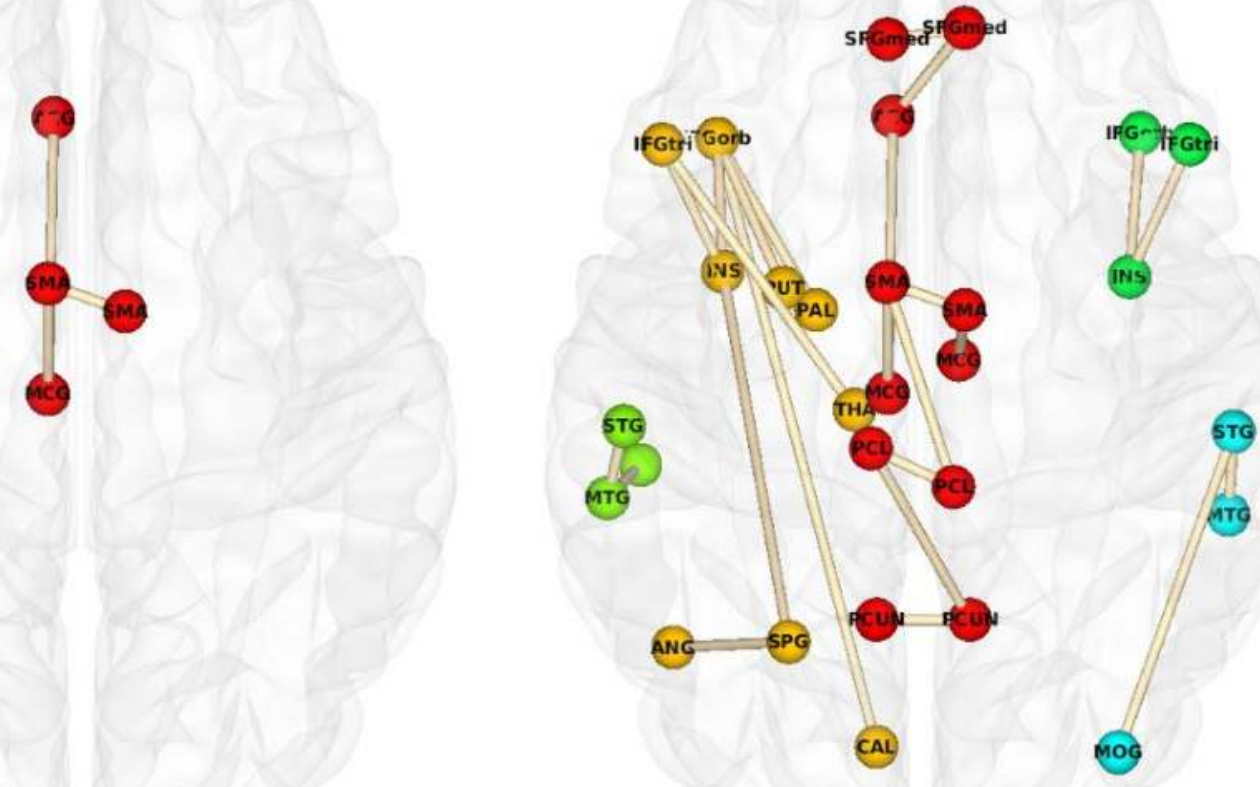

Figure 3: Topological clusters significantly associated with apathy in regional network analyses. A) Cluster that differed when comparing the apathy group to all other groups.

B) Clusters significantly associated with apathy in all subjects, while controlling for depression.

\section{Discussion}

Our study used network analysis to examine the relationship between white matter networks and apathy in patients with SVD. We found that network measures mediated the relationship between SVD markers and apathy, that patients with apathy, but not depression, had reduced whole brain measures of network density and efficiency, and that this disruption could be localised to specific structural subnetworks, which included parietal-premotor, frontostriatal, and occipitotemporal connections. Connectivity in premotor regions has been found to be associated with voluntary movement, while frontrostriatal circuitry is important for rewardbased decision-making. Our study suggests that damage to these white matter networks leads to a failure to initiate behaviour, manifesting clinically as apathy. 